\title{
Artificial Intelligence Tools for Enhancing Customer Experience
}

\author{
Joshi Sujata, Das Aniket, Matta Mahasingh
}

\begin{abstract}
Customers are the key to any business and the major challenge for any established business is retaining an existing customer and acquiring a new customer. One of the many ways to reduce the churn rate and increase customer retention is to improve the customer experience. As businesses are growing, their customer base is also increasing. Each and every customer is different and needs different kind of motivators to engage with the business and hence we need to understand each and every customer uniquely. Artificial Intelligence tools can blend the gap between the business and the client, creating enormous information that can prompt further comprehension of the client's preferences. Understanding these artificial intelligence tools and how these tools can assist organizations with retaining clients and help them give better involvement to their clients is significant. However, in academic research this significant research area stays under-focussed. Hence this study tries to address this gap by proposing a conceptual model for understanding how the Artificial Intelligence tools are can help in enhancing customer experience. The narrative literature review approach has been adopted for conceptualization of the model. The study provides implications to practitioners for designing and developing AI tools such that they enhance customer experience, to managers for designing the information technology strategy of their companies, to academicians as it helps explore new technologies in the marketing domain and to the society as it will help improve customer experience thereby leading to customer satisfaction.
\end{abstract}

Keywords- Artificial Intelligence, Big Data, Customer Experience, Personalization. Service Quality, Hassle free service.

\section{INTRODUCTION}

In the current digitized world, the quantity of information created by human beings as well as machines exceeds the ability of human beings with respect to absorption, interpretation, and complicated decision making based on that information. AI forms the basis for all computer learning and is the way forward for all advanced decision making. Britannica defines Artificial Intelligence as the ability of a digital computer or computer-controlled robot to perform tasks commonly associated with intelligent beings.' [1] Artificial intelligence can also be defined as "the simulation of human intelligence processes by machines, especially computer systems. These processes include learning (the acquisition of information and rules for using the information), reasoning (using rules to reach approximate or definite conclusio0ns) and self-correction". [2] The artificial intelligence needs to be engineered for

Revised Manuscript Received on July 10, 2019.

Joshi Sujata, Telecom Management, constituent of Symbiosis International (Deemed University), Pune, India (sjoshi@sitm.ac.in)

Das Aniket, Student Symbiosis Institute of Telecom Management, constituent of Symbiosis International (Deemed University) Pune, India.(das1820@sitm.ac.in)

Matta Mahasingh, Student Symbiosis Institute of Telecom Management, constituent of Symbiosis International (Deemed University) Pune, India (mahasingh.matta1820@sitm.ac.in) traits such as knowledge, reasoning, problem solving, perception, learning, planning and ability to solve problem. [3] The real task that lies ahead for the developers is to create A.I. that's capable of learning, thinking, and feeling without input from a human. This type of independent A.I. will be capable of making choices on its own, and can be considered really smart. [4]. Artificial Intelligence is dramatically redefining not only markets but also how marketers develop an understanding of customers, brands, markets segments and create and improve customer experiences. [5] Since technology has become the driving force of marketing, the developments in the area of artificial intelligence and its implications in the field of customer experience cannot be ignored. Artificial Intelligence helps to anticipate customer's next move and improve the customer journey. [6]. While the need for understanding of AI in customer experience has gained widespread interest, very few studies are available in academic literature regarding the same. Hence, this study tries to address this gap in literature by trying to achieve an understanding the long-term implications of AI in marketing. To achieve this objective the study focusses on use of Artificial Intelligence to provide better personalization, quality of service and hasslefree service which are important precursors to providing an enhanced customer experience.

\section{LITERATURE REVIEW}

Several papers have been published by different authors and technology related websites pertaining to Artificial Intelligence in marketing and some papers were focused on Customer Experience such as the paper by Gacanin and Wagner (2019) [7], which talks about the CEM components and the challenges with respect to the operator and the business requirement. The paper provides an overview of the path towards autonomous CEM framework and sets groundwork for future enhancements. The paper by Garcia (2018) [8] talks about the benefit of applying Data Driven Virtual Assistants and Artificial Intelligence for enhancing customer experience when managing Telecommunication services. This paper helps us identify the benefits of both the user as well as the Telecom organization that implement a Data driven Virtual Assistant. The papers by Wilson \& Daugherty [9] talks about how AI is helping humans reach conclusions through the process that are opaque or require human experts in field to explain the behavior to non-expert

Published By:

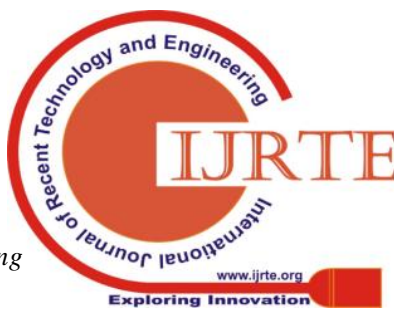


users. The paper in general talks about how training of chatbots is important and with par how using the chatbots data by the employees can be used by the employees to improve the Customer Experience. The paper by Bäckström and H. Larsson (2018) [10] talks about how the ecommerce companies are working toward adaptation of AI into companies CRM systems. The paper also explains how AI within CRM could be beneficial as a tool on the Global market. The paper by T. Brill (2018) [11] majorly talks about using analysis of the relative importance of model constructs and tries to map customer satisfaction with digital assistants with the help of primary data.

This academic paper focuses on developing a conceptual framework for understanding how the Artificial Intelligence tools are can help in enhancing customer experience.

\section{THEORETICAL FRAMEWORK AND RESEARCH QUESTIONS}

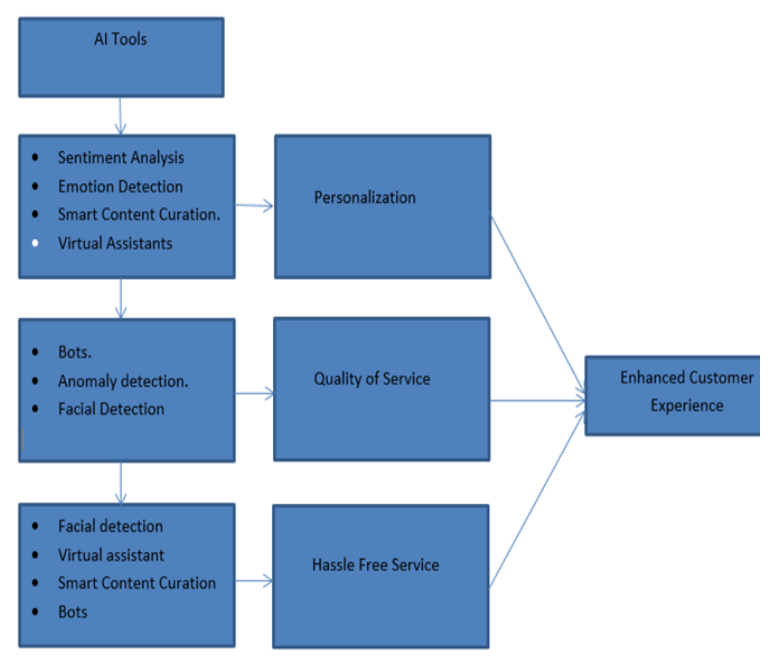

Figure 1. Conceptual Model

Figure 1 demonstrates a theoretical model dependent on sources referenced in the former area. Through this conceptual model we propose that AI tools lead to Personalization, Improved service quality and Hassle free service which in turn lead to enhanced customer experience. Hence through this study, the researchers are trying to address the following research questions:

1. Do AI tools like Sentiment analysis, Emotional detection, Smart content curation and Virtual assistants lead to Personalization?

2. Do AI tools like Bots, Anomaly detection and Facial detection lead to Improved Service Quality?

3. Do AI tools like Facial detection, Virtual assistant, Smart Content Curation and Bots lead to Hassle-free services?

4. Does Personalization, Improved service quality, Hassle-free services using AI tools lead to Enhanced Customer Experience?

\section{RESULTS, ANALYSIS AND DISCUSSIONS}

\subsection{A.I tools leads to Personalization:}

Digital personalization is the process of creating customized experiences for one's customers. Personalization allows visitors with unique experiences suited to the customer's needs and requirements. [12] Personalization is different from customization. Personalization is achieved when a system creates an experience based on the consumer's previous behaviors whereas, customization is realized when the customer manually make changes so that they can achieve their preferred experience. [13] Examples of offline personalization can be seen when waiters often greet their regular customers and also remember their usual order. Similarly, digital examples can be seen in top brands such as Amazon, Starbucks etc., where the companies use the purchase history and location data to get as many customer insights as possible. Netflix uses an algorithm that suggests what the customer might want to watch next based on their viewing history. [14]

\subsubsection{Sentiment Analysis and Personalization}

Sentiment analysis is the computerized procedure of understanding a supposition about a given subject from composed or spoken language. Opinion examination is one of the tools that help in comprehending the information that is created each day. Sentiment analysis can extract different attributes from a statement such as polarity - whether the speaker is expressing a positive or a negative view, subject what is being talked about, opinion holder - The person or entity expressing the view. This kind of outcome is possible due to natural language processing (NLP). Sentiment analysis can be done at different levels - document, sentence and sub-sentence. Also, sentiment analysis can be used for emotion detection. [15]. Sentiment analysis is done by using techniques such as - Naïve Bays algorithm, linear regression, support vector machines and deep learning. [15] Sentiment analysis is a useful application of personalization. One of the attractive points about sentiment analysis is that when the system is subjected to more data, it trains itself and the analysis becomes more and more accurate. Hence, when it comes to attaining accurate information about the customer's needs, sentiment analysis becomes a powerful tool. Sentiment analysis can be used from macro campaign level to micro wording of a landing page. Sentiment analysis allows you to fine tune your message. [16].Facebook uses sentiment analysis to understand the intent of the users who are using the website. Sentiment analysis also allows Facebook to understand what the people feel about the additions/removal of features that are done via regular updates. [17]

\subsubsection{Emotion detection and Personalization}

Emotion recognition is a procedure wherein a program is utilized to "read" the feelings on a human face utilizing advanced image processing. Organizations have been exploring different avenues regarding joining modern calculations with image processing techniques to see increasingly about how an individual is feeling. [18] This when used in conjunction with sentiment analysis can help in providing useful insights for an organization. Emotion detection is used by many Fortune 500 companies such as Disney, Kellogg, and Unilever etc. Kellogg shows multiple versions of the same ad in order to test the audience.

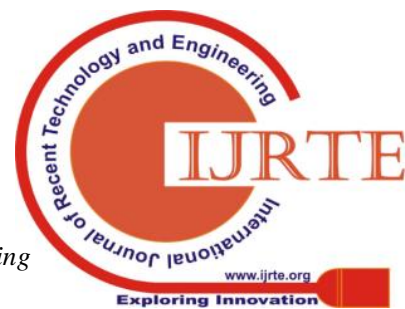


Kellogg then uses the ad that had the most customer engagement or the ad with which the emotions of the customers lined up that the company was hoping to portray. Disney uses emotion AI for market research so that it can determine how audiences enjoy its movies. Disney used infrared cameras to capture audience reactions while they were watching 'The Jungle Book' and 'Star Wars: The enough that after a few minutes of tracking the facial behavior, it was able to predict whether the viewer will smile or laugh in the coming scenes. This also creates a more accurate image of the audience response. [19]

\subsubsection{Smart Content Curation and Personalization}

Content promoting is done as such that one can control the guests of their site or advanced resources, draw in them, transform them into paying clients and hold them. [20] Content curation is one of the content marketing strategies. Content curation can be defined as - "the act of discovering, gathering, and presenting digital content that surrounds specific subject matter." "Content curation is different from the content is amassed from various sources and organized in a specific manner". [21] There are many tools for online content curation such as Buzzsumo, Twitter Lists, Pocket etc. [22] With the help of artificial intelligence, it is now possible to auto generate marketing emails at a large scale, with each recipient receiving their own personalized curated content based on their interests. Vestorly is an A.I enabled content marketing platform, which can do this. Vestorly or other such platforms have an A.I powered search engine core which crawls through websites, tweets, stories, videos etc. in real time and aggregates and organizes them. When a user imports some personal data, the system automatically offers content which it seems more likely to be engaging with the user. The recommendations are based on the personal data and the contacts that the user seems to have. Vestorly is a system that is built for email personalization. [23]

\subsubsection{Virtual Assistants and Personalization}

Virtual assistant is a software agent that can play out specific undertakings or services in the interest of an individual dependent on a blend of client info and area. They are capable of performing complicated tasks with complete voice support. Virtual Assistants perform functions in real time such as calendar and meeting reminder, identify interesting landmarks when the user is near one, have a natural conversation, integrate with other apps such as play music or show specific news etc. [24]. Virtual assistants are powered by artificial intelligence which helps them in performing several complicated tasks and are trained in natural language processing so that they understand instructions as well as provide results fluently. Google Assistant, Siri, Google Now, Bixby, Cortana, Amazon Echo are some of the top virtual assistants.

Proposition 1: AI tools like Sentiment analysis, Emotional detection, Smart content curation and Virtual assistants lead to Personalization.

\footnotetext{
4.2 Artificial Intelligence leads to enhanced service quality
} Force Awakens'. The emotion algorithm was powerful content marketing, in which content is generated. Instead,

Maintaining an old customer is much cheaper than acquiring a new customer. The old customers are valuable to any organization. According to a report acquiring a new customer costs the organization five times more amount than retaining their loyal customer and increasing customer retention by $5 \%$ can help the organization in improving their bottom-line by $25-95 \%$ as the case may be [25]. The clients are expanding at an exponential rate hence keeping up legitimate service quality is particularly significant. This is the place artificial intelligence becomes an integral factor. Artificial Intelligence tools, for example, bots, inconsistency discovery and facial recognition help the association keep up a superior nature of service quality and thus improving the customer experience.

\subsubsection{Employee Facing Bots and Enhanced service quality}

Virtual assistants or bots can be sent to help the employees, for example, by giving brisk answer formats, directing quicker ventures of the inner learning stage, or supporting another operational advance. The bots have a calculation that can break down the patterns and make snappy formats for answering the clients and in case the solution is not handy with the employee the bot can find the required knowledge article for the customer-facing employee [26].

Microsoft AI offers employee-facing bots as a piece of their Dynamics 356 solution. Inward offices at Microsoft, just as HP and Macy's are as of now utilizing this innovation to improve by and large consumer loyalty by taking care of more requests in a shorter amount of time. [27]. While these bots doesn't interact with the customer but can help in improving the customer experience by decreasing the average resolution time for the support time.

\subsubsection{Anomaly detection and Enhanced service quality}

Anomaly detection is recognition of rare items, occurrence or observation that raises suspicion by differing significantly from the major chunk of the data. Typically the anomalous items will convey some kind of problem such as frauds, data breach etc. Anomalies are coined as outliers, noise, deviation and exception [28]. Artificial Intelligence tools harnesses predictive analytics and end to end machine learning techniques to automatically identify anomalies by continuously learning from the past data. This helps in avoiding false alerts and helps in prioritizing the problems automatically [29] Netflix one of the most prominent Over the Top service provider is using Anomaly detection in full swing. They use anomaly detection for the millions of transitions that have a failure from the bank end and they use in the customer signup. It has helped in improved turnaround time for the employees of Netflix and improved quality of service for their existing customer by not adding fraudulent customers to their system. [30]. Anomaly detection can help in minimizing the potential threats to any organization and refraining fraudulent users from using the company's services thus improve the bandwidth per user of the existing customer, thus improving the service quality. 


\subsubsection{Facial Recognition and enhanced service quality}

Facial recognition is a mechanism of recognizing human faces through technology. It uses biometrics to map features from the image captured by the cameras and then compares the data in its existing data base. It is used to verify personal Identity [31]. In offline retail store, there are huge queues of shoppers and the shoppers are not willing to wait for a very long time, degrading the overall customer experience. Walmart, the retail giant of United States has rolled out facial recognition for autonomous payment, finding dissatisfied customers and for reducing any kind of fraudulent activities in stores that is helping them provide better service to their existing customer. [32]. Facial recognition can help in autonomous checkout at retail stores, thus the customers need not face any kind of queue while billing, it helps to find out the dissatisfied customers when they are shopping and finding out the reason for such behavior and reducing any kind of potential fraud in their stores, thus improving the quality of service provided by the organization.

Proposition 2: AI tools like Bots, Anomaly detection and Facial detection lead to Improved Service Quality.

\subsection{AI tools lead to hassle-free service}

Hassle-free service is one of the most important factors when it comes to providing services to a customer. American Marketing Association defines service marketing as "the activities, benefits or satisfactions which are offered for sale are provided in connection with the sale of goods." For example, when a customer pays for a night at a hotel, he is not only paying for the accommodation but also for a homely experience and expects the services to be smooth. Providing problem-free services has many benefits such as recurring sales, improved reviews, word-of-mouth publicity etc. [33]. With the advent of technology and the rise of A.I, it has become easier to provide better services to the consumers and manage their needs more efficiently. Artificial Intelligence can help marketing team keep a track of all the information they are dealing with on a regular basis and in real-time. These tools work in the form of analytical tools, chatbots, assistants etc. Analytical tools such as Albert, Nudge, and Sensai etc. provide means to prepare tailor-made campaigns for their customers. Tools such as Acrolinx, Kenshoo help in content management and ad-optimization. There are many other tools which help marketing, social media management, workflow automation etc. [34]

\subsubsection{Facial detection leads to hassle-free service}

Facial recognition is a biometric system that is used to identify and authenticate a person by just using his/her facial identity. The facial features of the person are mapped and stored in the database and when a person tries to authenticate themselves, the mapped image from the database is used for reference. Facial recognition software can tell the difference between a real and a fake image. It is more accurate than humans at identifying faces, it is quick and it is difficult to hack. One of the applications of facial recognition is its use at the airports. Airports generally use this type of technology for security purposes. Earlier, marketing team in providing better customer service, email

physical verification was required at the immigration desk at an airport. But, nowadays, Airports have started using facial recognition for immigration also. An array of cameras use facial recognition to identify the person and a scanner is used to scan the person's passport. This process is faster. Airports in the United States of America, Australia etc. have started using these techniques. With the help of facial recognition, security checks become a hassle-free process. The technology is highly secure, accurate and fast. [35]

\subsubsection{Virtual assistant leads to hassle-free service}

Virtual assistants have evolved into conversation capable application since the early days of Apple's Siri. Mobile phone services can now be used with using only one's voice by using assistants such as Siri, Cortana or Google Assistant. These virtual assistants can perform tasks such as call up people, send or read texts, find information, give you directions etc. [36]. Another example of virtual assistant providing hassle-free service is that of "Elon" - which a virtual assistant for Tesla cars is. The users of these virtual assistants can impart by means of voice and visit messages remotely to perform activities, for example, locking/opening or leaving the vehicle. The user can also get car health details and makes the car an entity with which the user can communicate to bringing in a personal connection rather than communicating with an inanimate object. [37]

\subsubsection{Smart Content Curation leads to hassle-free service}

Content curation is a marketing technique that provides relatable content to users. This helps in customer engagement. With better curation techniques this has become easier. Also, the user starts expecting quality content from the curators be it - news, fashion tips, music, lifestyle recommendations etc. If a curator wants increase customer engagement then the curator needs to provide relevant content to the users. As the number of users increase, the need for automation rises in order to provide hassle-free service. Tools work in an incremental process where the content is first collaborated, it is then curated, analytics is applied to the media platform in the third stage and finally engagement automation is done where the content is delivered to the consumers. Pocket, Learnist Tweets, Kuratur, iFlow are some of the top content curating apps. [38]

\subsubsection{Bots lead to hassle-free service}

A Chabot is a man-made artificial intelligence tool which leads a discussion with an individual. Chabot's have turned out to be mainstream because of progression in innovation and AI. Due to this advancement, chatbots have evolved such that they can not only communicate with humans but also understand complicated queries. Due to this, chatbots have started replacing human customer service representatives. Chabot's have become so sophisticated that $45 \%$ of end users prefer communicating with a Chabot rather than a human representative. Thus, we can say that chatbots help in providing better customer service. [39]. End users want immediate resolution to their issues be it day or

Engineering \& Sciences Publication 
night and it becomes difficult for an organization to cater to a huge audience on a personal basis. The turnaround time for such an interaction is very high and also many a times, the customer never connects with the human representative due to time constraints. Hence, in such a scenario chatbots make it easier for an organization to solve user queries and issues. [40].

Proposition 3: AI tools like Facial detection, Virtual assistant, Smart Content Csuration and Bots lead to Hasslefree services.

\subsection{Customer Experience}

Customer Experience is what the customers of that brand perceives via all interaction across the lifecycle of the customer. Gartner defines "Customer experience is the perception of the customer and their feelings related to the employees, system, channel or product" [41].

\subsubsection{How personalization leads to Customer Experience}

Clients are totally responsible for the associations with the brands they partner. In this hypercompetitive market items and administrations are very little differentiable. The customized experience is the discussion of great importance. Certain organizations are conveying a custom-made encounter to their client and that is helping them drive higher incomes for their organization. As indicated by a study, $49 \%$ of the general population purchase an item that they mean to purchase however winds up purchasing due to personalization [42]. Artificial Intelligence tools such as Emotional detection, Sentimental analysis provides a customized feel when they interact with the customer providing the customer with instant satisfaction. Personalization helps in providing a better association with the brand and it helps in improving the customer experience.

\subsubsection{How enhanced service quality leads to Customer experience.}

In this hypercompetitive environment organization's need to find a perfect way to get associated with their existing customers and is very much evitable that customers while using the product needs some kind of assistance. Providing them with the required assistance and by reducing the turnaround time is very much required in today's business. Artificial Intelligence tools such as Chabot's helps reduce the burden of the customer care executives by continuously learning from the conversation with the customers. Employee-facing bots help in reducing the turnaround time for the customer care executive by helping them find the appropriate resources from the knowledge base automatically. Customers are not willing to remain unattended by the organizations and their requirements are getting their problems solved immediately. Businesses need to provide top-notch service quality to their customer for retaining the customer and provide them with enhanced customer experience.

\subsubsection{How hassle-free service leads to Customer experience}

The business of every scale realized that delivering premium customer experience is the key to increase customer retentions and attracting new customers, ultimately boosting the bottom line for the organization. The differential factor for a better organization is providing extraordinary customer experience to its customer [43]. Whenever a customer visits a supermarket or any retail store, they need to stand in a long queue at the checkout counters for checkout, this provides the customer with negative customer experience [44] The customers standing in the queue becomes restless and sometimes doesn't want to stand in that queue for checkouts. Artificial Intelligence tools such as facial detections detect the customer and selfcheckouts can be done to provide the customers with superior customer experience. There are many AI tools such as automated content creation, bots etc. that helps in providing the customer with a hassle-free experience, that in return helps in creating a better customer experience.

Proposition 4: Personalization, Improved service quality, Hassle-free services using AI tools lead to Enhanced Customer Experience.

\section{RESEARCH IMPLICATIONS AND FUTURE RESEARCH DIRECTIONS \& RESULTS}

The proposed model shows that Artificial Intelligence tools such as sentiment analysis, emotion detection, virtual assistants, chatbots, and content curation lead to better personalization, quality of service and hassle-free service and in turn provides an enhanced customer experience. Positive experiences lead to feeling of satisfaction and better trust on the brand, depending on the degree of involvement of the customer and the perceived brand value. Customers who experienced personalization, smooth and superior quality of service would have a more loyalty towards the brand. These customers will be more inclined to refer the brand to other consumers, provide feedback on improvements etc. The study provides implications to practitioners for designing and developing AI tools such that they enhance customer experience, to managers for designing the information technology strategy of their companies, to academicians as it helps explore new technologies in the marketing domain and to the society as it will help improve customer experience thereby leading to customer satisfaction.

The conceptual model needs empirical verification. Hence an empirical study can be undertaken to test the propositions stated by this study. Further, there is a need for research in the cross disciplinary areas to identify the important features that will enhance the customer experience with the help of technology. As customer experience is one of the important perspective for any business these kind of research can help client facing manager making the customer experience more pleasant for consumers.

\section{REFERENCES}

1. B. Copeland, "Artificial intelligence," Britannica, 09 May 2019 [Online]. Available: https://www.britannica.com/technology/artificialintelligence. [Accessed 13 May 2019].

2. M. Rouse, "What is AI," SearchEnterpriseAI, August 2018. [Online]. Available: https://searchenterpriseai.techtarget.com/definition/AIArtificial-Intelligence [Accessed 13 May 2019] 
3. "What is Artificial Intelligence," Technopedia, [Online]. Available: https://www.techopedia.com/definition/190/artificial-intelligence-ai [Accessed 13 May 2019].

4. R. V. Loon, "Google Deepmind," Digital Doughnut, 23 Apr 2018. [Online]. https://www.digitaldoughnut.com/articles/2018/april/googledeepmind-the-importance-of-ai. [Accessed 13 May 2019].

5. P. Kushmaro, "How AI is reshaping marketing," CIO, 04 Sept 2018. reshaping-marketing.html. [Accessed 13 May 2019].

6. L. Tjepkema, "What Is Artificial Intelligence Marketing?," Emarsys, [Online Available: https://www.emarsys.com/resources/blog/artificial-intelligencemarketing-solutions/. [Accessed 13 May 2019].

7. H. Gacanin and M. Wagner, "Artificial Intelligence Paradigm for Customer Experience Management in Next-Generation Networks: Challenges and Perspectives,," IEEE Network, vol. 33, no. 2, pp. 188 194, March/April 2019.

8. M. P. Garcia, "The potential of Data-Driven Virtual Assistants to enhance Customer Experience in the Telecommunications Industry," HFD, vol. 7, no. 13, pp. 61-72, July 2018.

9. H. J. Wilson \&. P. R. Daugherty, "Collaborative Intelligence: Humans and AI Are Joining Forces," July 2018. [Online]. Available: https://hbr.org/2018/07/collaborative-intelligence-humans-and-ai-arejoining-forces. [Accessed 13 May 2019].

10. A. Bäckström and H. Larsson, 'Is There Suh A Thing As Too Much Intelligence?: A qualitative study exploring how Born Global ecommerce companies are working towards adopting Artificial Intelligence into their Customer Relationship Management Systems', Dissertation, 2018

11. Brill, Thomas M., "Siri, Alexa, and Other Digital Assistants: A Study of Customer Satisfaction With Artificial Intelligence Applications" (2018). Electronic Dissertations \& $\quad$ Theses. 1. http://digitalcommons.udallas.edu/edt/1

12. Optimizely, "Website Personalization," Optimizely, [Online] Available: https://www.optimizely.com/optimizationglossary/website-personalization/. [Accessed 13 May 2019].

13. P. Davis, "What is the Difference Between Personalization and Customization?," towerdata, 5 Nov 2018. [Online]. Available: https://www.towerdata.com/blog/what-is-the-difference-betweenpersonalization-and-customization. [Accessed 13 May 2019].

14. L. Keane, "10 Brilliant Examples of Personalized Marketing and Why They Worked," globalwebindex, 11 Apr 2019. [Online]. Available: https://blog.globalwebindex.com/marketing/personalized-marketingworks/. [Accessed 13 May 2019].

15. MonkeyLearn, "Sentiment Analysis," MonkeyLearn, [Online] Available: https://monkeylearn.com/sentiment-analysis/. [Accessed 13 May 2019].

16. C. Walther, "Sentiment Analysis in Marketing: What Are You Waiting For?," CMSWiRE, 2 Jan 2019. [Online]. Available: https://www.cmswire.com/digital-marketing/sentiment-analysis-inmarketing-what-are-you-waiting-for/. [Accessed 13 May 2019].

17. S. Gupta, "Sentiment Analysis: Concept, Analysis and Applications," Towards Data Science, 7 Jan 2018. [Online]. Available: https://towardsdatascience.com/sentiment-analysis-concept-analysisand-applications-6c94d6f58c17. [Accessed 13 May 2019].

18. Technopedia, "Emotion Recognition," Technopedia, [Online] Available: https://www.techopedia.com/definition/30819/emotionrecognition. [Accessed 13 May 2019].

19. N. Gilliland, "How brands are using emotion-detection technology," Econsultancy, 13 Sept 2018. [Online]. Available: https://econsultancy.com/how-brands-are-using-emotion-detectiontechnology/. [Accessed 13 May 2019].

20. "7 content assets that are integral to your content marketing strategy," Smart Insights, 04 Jan 2019. [Online]. Available: https://www.smartinsights.com/content-management/contentmarketing-strategy/7-content-assets-integral-your-content-marketingstrategy/. [Accessed 13 May 2019].

21. E. Mullan, "What is Content Curation?," EContent, 30 Nov 2011. Online

Available: http://www.econtentmag.com/Articles/Resources/DefiningEContent/What-is-Content-Curation-79167.html. [Accessed 13 May 2019].

22. T. Clarke, "The Complete Guide to Content Curation: Tools, Tips, Ideas," Hootsuite, 24 Oct 2018. [Online]. Available: https://blog.hootsuite.com/beginners-guide-to-content-curation/. [Accessed 13 May 2019]. [Online]. Available: https://www.cio.com/article/3302739/how-ai-is-

23. M. Unemyr, "SEND ONE-TO-ONE PERSONALIZED CURATED CONTENT AT SCALE WITH AI," Unemyr, 27 Mar 2018. [Online] Available: https://www.unemyr.com/ai-curated-content-vestorly/. [Accessed 13 May 2019].

24. Imanuel, "Top 22 Intelligent Personal Assistants or Automated Personal Assisstants," Pat Research, [Online]. Available: https://www.predictiveanalyticstoday.com/top-intelligent-personalassistants-automated-personal-assistants/. [Accessed 13 May 2019].

25. T. Landis, "Outbound Engine," 2 Feb 2019. [Online]. Available: https://www.outboundengine.com/blog/customer-retentionmarketing-vs-customer-acquisition-marketing/. [Accessed 13 May 2019].

26. Ibenta, "5 ways to use chatbots for internal Employees," inbenta, 2 July 2018. [Online]. Available: https://www.inbenta.com/en/blog/5chatbots-internal-employees/. [Accessed 13 May 2019].

27. Dynamics 365, "Microsoft Dynamics 36 AI," Microsoft, [Online]. Available: https://dynamics.microsoft.com/en-us/ai/overview/ [Accessed 13 May 2019].

28. Technopedia, "Anomaly Detection," Technopedia, [Online] Available: https://www.techopedia.com/definition/30297/anomalydetection. [Accessed 13 May 2019].

29. Dynatrace, "Anomaly Detection," dynatrace, [Online]. Available: https://www.dynatrace.com/platform/artificial-intelligence/anomalydetection/. [Accessed 13 May 2019].

30. J. Wong, C. Colburn, E. Meeks and S. Vedaraman, "RAD—Outlier Detection on Big Data," Medium, 19 Feb 2015. [Online]. Available: https://medium.com/netflix-techblog/rad-outlier-detection-on-bigdata-d6b0494371cc. [Accessed 13 May 2019].

31. S. Symanovich, "How does facial recognition work?," Norton, [Online]. Available: https://us.norton.com/internetsecurity-iot-howfacial-recognition-software-works.html. [Accessed 13 May 2019].

32. D. O'Shea, "Report: Walmart developing facial-recognition tech," Retail Dive, 19 July 2017. [Online]. Available: https://www.retaildive.com/news/report-walmart-developing-facialrecognition-tech/447478/. [Accessed 13 May 2019].

33. P. Chetty, "Concept of service marketing," Project Guru, 30 Oct 2010 [Online]. Available: https://www.projectguru.in/publications/service marketing/. [Accessed 13 May 2019]

34. A. Cross, "50 Must-Have AI Software Tools For Marketers," NGDATA, 6 Aug 2016. [Online]. Available: https://www.ngdata.com/must-have-ai-software-tools-formarketers/\#sect2. [Accessed 13 May 2019].

35. "Facial Recognition Software at Airports to ease congestion," InnefuLabs, [Online]. Available: https://www.innefu.com/blog/facialrecognition-software/. [Accessed 13 May 2019].

36. L. M. Martin, "23 Best Virtual Assistant Software Tools to Optimize Productivity," Time Doctor, [Online]. Available: https://biz30.timedoctor.com/virtual-assistantsoftware/\#Virtual\%20Assistant\%20Software. [Accessed 13 May 2019].

37. W. A. M. A. Asad Ullah, "Smart Automation, Customer Experience and Customer Engagement in Electric Vehicles," MDPI, vol. 10, no. 1350, p. 11, Apr 2018.

38. E. Garcia, "Curated List of 16 ABSOLUTELY FREE Content Curation Tools," David Moceri, 20 July 2018. [Online]. Available: https://davidmoceri.com/curated-list-16-absolutely-free-contentcuration-tools/. [Accessed 13 May 2019].

39. S. Suthar, "Top 11 Chatbot Trends That Will Help to Grow Your Business in 2019," Acquire.io, 22 Apr 2019. [Online]. Available: https://acquire.io/blog/chatbots-trends/. [Accessed 13 May 2019].

40. L. Vishnoi, "10 Chatbot Benefits: A Business Can’t Afford to Miss," Acquire.io, 23 Jun 2018. [Online]. Available: https://acquire.io/blog/chatbot-benefits-for-business/. [Accessed 13 May 2019].

41. Gartner, "Customer Experience," Gartner, [Online]. Available: https://www.gartner.com/it-glossary/customer-experience. [Accessed 13 May 2019]

42. S. Hyken, "Personalized Customer Experience Increases Revenue And Loyalty," Forbes, 29 Oct 2017. [Online]. Available: https://www.forbes.com/sites/shephyken/2017/10/29/personalizedcustomer-experience-increases-revenue-and-loyalty/\#58405d234bd6 [Accessed 13 May 2019]. 
43. M. Galetto, "Top Customer Experience Strategy Resources: 50 Articles, Guides, Presentations, and More to Help You Refine and Execute Your Customer Experience Strategy," NGDATA, 13 Dec 2017. [Online]. Available: https://www.ngdata.com/customerexperience-strategy-resources. [Accessed 13 May 2019].

44. K. Tsernov, "Long Waiting Times Cost You Sales," Qminder, [Online]. Available: https://www.qminder.com/long-waiting-timessales/.. [Accessed 13 May 2019]. 